\title{
Symbolic Analysis of 24h Holter Heart Period Variability Series: Comparison between Normal and Heart Failure Patients
}

\author{
A Porta ${ }^{1}$, G D'Addio $^{2}$, GD Pinna ${ }^{3}$, R Maestri $^{3}$, T Gnecchi-Ruscone ${ }^{4}$, \\ R Furlan ${ }^{4}, \mathrm{~N}_{\text {Montano }}{ }^{4}, \mathrm{~S}_{\text {Guzzetti }}{ }^{4}, \mathrm{~A} \mathrm{Malliani}^{4}$ \\ ${ }^{1}$ DiSP LITA di Vialba, Universita' di Milano, Milan, Italy \\ ${ }^{2} \mathrm{~S}$ Maugeri Foundation, IRCCS, Rehabilitation Institute of Telese, Italy \\ ${ }^{3}$ S Maugeri Foundation, IRCCS, Rehabilitation Institute of Montescano, Italy \\ ${ }^{4}$ DiSC “Luigi Sacco", Universita' degli Studi di Milano, Milano, Italy
}

\begin{abstract}
This study proposes an application of symbolic analysis to beat-to-beat heart rate variability data derived from $24 \mathrm{~h}$ Holter recordings both in healthy and heart failure populations. Heart rate series are transformed in a sequence of six symbols via a uniform quantization procedure. Symbols are grouped in patterns lasting three cardiac cycles and these patterns are grouped in few (four) families without any loss. The rate of occurrence of these families are found useful to distinguish two physiological conditions characterised by a different status of the autonomic nervous system (i.e. day-time and nigh-time) in healthy subjects and to discriminate between healthy and pathological populations. Indexes derived from symbolic analysis deserve to be added to traditional time and frequency domain parameters in standard analysis of heart rate variability obtained from $24 \mathrm{~h}$ Holter recordings and tested over larger databases.
\end{abstract}

\section{Introduction}

Symbolic analysis applied to heart rate variability is providing new parameters independent of those derived from time and frequency domains and helpful in interpreting the encoded physiological information [1-4]. Voss et al [1] demonstrated that the addition of indexes originated from symbolic analysis to traditional time and frequency domain indexes improves the accuracy of risk stratification after myocardial infarction. Wessel et al [2] found that parameters from symbolic analysis discriminate chronic heart failure patients with high risk to develop life-threatening arrhythmias from those with marginal risk, while time and frequency domain parameters cannot separate the two populations. Guzzetti et al [3] showed that symbolic analysis in healthy subjects is sensible to the activation of the autonomic nervous system as much as power spectral analysis with the additional possibility to permit the detection of concomitant activation of sympathetic and parasympathetic autonomic nervous systems.

The aim of this study is to propose an application of symbolic analysis to heart rate variability series obtained from standard 24h Holter recordings. In this application we have considered healthy subjects and heart failure patients to verify whether symbolic analysis can detect in normal population the well-known differences between day-time and night-time and can discriminate the two populations.

The core of the approach lies in the application of the previously published symbolic analysis method [4] searching for the rates of occurrence of specific short patterns (i.e. sequences of symbols lasting 3 cardiac cycles). This approach, designed to reliably work on short beat-to-beat series (about 300 beats), is iteratively applied to $24 \mathrm{~h}$ Holter heart rate variability recordings.

\section{Symbolic analysis}

Given the series $x=\{x(i), i=1, \ldots, N\}$, where $i$ is the progressive sample counter and $\mathrm{N}$ is the series length, $\mathrm{x}$ is transformed in a sequence of symbols using a coarse graining approach based on a uniform quantization procedure [4]. The full range of the series is spread over $\xi$ symbols with a resolution of $\left(\mathrm{x}_{\max }-\mathrm{x}_{\min }\right) / \xi$, where $\mathrm{x}_{\max }$ and $\mathrm{x}_{\min }$ are the maximum and the minimum of the series. After quantization $\mathrm{x}$ becomes a sequence $\mathrm{x}_{\xi}=\left\{\mathrm{x}_{\xi}(\mathrm{i})\right.$, $\mathrm{i}=1, \ldots, \mathrm{N}\}$ of integer values ranging from 0 to $\xi-1$. The series $x_{\xi}$ is transformed in a sequence of patterns of $\mathrm{L}$ delayed samples, $\quad x_{\xi, L}=\left\{x_{\xi, L}(i), \quad i=L, \ldots, N\right\} \quad$ with $x_{\xi, L}(i)=\left(x_{\xi}(i), x_{\xi}(i-1), \ldots, x_{\xi}(i-L+1)\right)$ and the number of possible $x_{\xi, L}(i)$ is $\xi^{L}$. The rate of occurrence of each $x_{\xi, L}(i)$ can be calculated by dividing the number of times that $x_{\xi, L}(i)$ is found in $x_{\xi}$ by $\xi^{L}$ (i.e. the sample frequency of $\left.\mathrm{x}_{\xi, \mathrm{L}}(\mathrm{i})\right)$. As $\xi^{\mathrm{L}}$ grows very rapidly with $\mathrm{L}$ and $\xi$, both parameters have to be small, thus limiting the length of 
the pattern $\mathrm{L}$ and the resolution of the coarse graining procedure. For applications over short data sequences (about 300 samples) the best compromise is $\xi=6$ and $\mathrm{L}=3$ [4], thus maintaining a pattern as long as possible without loosing too much resolution.

\section{Pattern classification}

With $\xi=6$ and $L=3$ the number of possible patterns are 216. Although this number is not high, following the changes in the rate of appearance of all these patterns may be difficult. Therefore, a procedure of redundancy reduction is applied and all the patterns are grouped without any loss into four families according to the number and types of variations from one symbol to the next one [4]. The pattern families are: i) pattern with no variation $(0 \mathrm{~V}$, all the symbols are equal, e.g. $(4,4,4)$ or $(0,0,0))$; ii) patterns with one variation ( $1 \mathrm{~V}$, two consecutive symbols are equal and the remaining one is different, e.g. $(1,3,3)$ or $(5,2,2))$; iii) patterns with two like variations $(2 \mathrm{LV}$, the three symbols form an ascending or descending ramp, e.g. $(2,3,5)$ or $(4,3,0))$; iv) patterns with two unlike variations ( $2 \mathrm{UV}$, the three symbols form a peak or a valley, e.g. $(2,4,3)$ or $(5,2,4))$. The rate of occurrence of these patterns is indicated as $0 \mathrm{~V} \%, 1 \mathrm{~V} \%$, $2 \mathrm{LV} \%$ and $2 \mathrm{UV} \%$ and evaluated over series of length $\mathrm{N}=300$.

\section{Protocol and data analysis}

We studied 12 normal $(\mathrm{N})$ healthy humans (age from 34 to 55) and 9 heart failure (HF) patients (NYHA class II and III, age from 36 to 68 , ejection fraction from $14 \%$ to $34 \%$ ) undergoing 24h Holter recordings (Oxford Medilog System). Only recordings with at least $50 \%$ of the analysed period in sinus rhythm, during both night-time and day-time has been considered. Twenty-four hour beat-to-beat RR series were preprocessed according to the following criteria: 1) RR intervals associated with single ectopic beats were replaced by their mean value; 2) artifacts and runs of tachycardic beats were replaced by $\mathrm{N}$ values equal to the mean $\mathrm{RR}$, in such a way that $\mathrm{N}^{*}$ mean $\mathrm{RR}$ was less than or equal to the substituted value; 3) RR values differing from the preceding one more than $30 \%$ (absolute value) were replaced as for artifacts. The mean RR was computed as a moving average centred on the beat to correct, with a buffer of \pm 3 beats labelled as normal. The $24 \mathrm{~h}$ heart rate variability series were analyzed during day-time (from 9:00 AM to 7:00 PM) and during night-time (from 0:00 to 5:00 AM). Pattern classification was applied iteratively over sequences of 300 cardiac beats with a $50 \%$ overlap. The median of the distributions of $0 \mathrm{~V} \%, 1 \mathrm{~V} \%, 2 \mathrm{LV} \%$ and $2 \mathrm{UV} \%$ was evaluated during the day-time and night-time. Results were reported as mean \pm standard deviation.
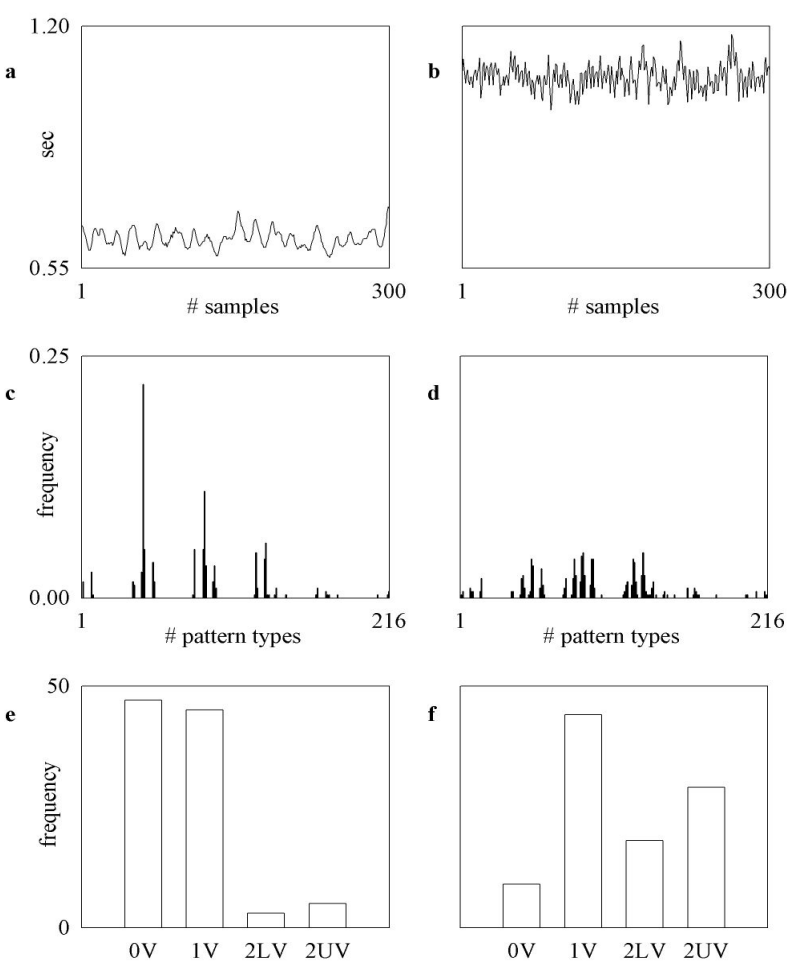

Figure 1. Example of RR series (300 beats) during daytime (a) in a $\mathrm{N}$ subject. Related distributions of all patterns and of the four pattern families are reported in (c) and (e) respectively. The same is shown for an example of RR series recorded at night-time $(b, d, f)$ in the same subject.

t-test for paired data was applied to check the significance of the differences between day-time and night-time in the same population. t-test for unpaired data was applied to verify whether the two populations (i.e. $\mathrm{N}$ and HF) were significantly different during the same experimental condition (day-time or night-time). A $\mathrm{p}<0.05$ was considered statistically significant.

\section{Results}

Fig.1 shows two examples of RR interval series lasting 300 cardiac beats extracted randomly from a $24 \mathrm{~h}$ heart rate variability series during day-time (Fig.1a) and nighttime (Fig.1b) in a $\mathrm{N}$ subject. The distributions of all the patterns derived from these two series appear to be very different. During day-time (Fig.1c) the distribution is characterised by a large amount of missing patterns and by the presence of few patterns that are more frequently detected than others. During night-time (Fig.1d) the number of missing patterns is decreased and the rate of occurrence of the existing patterns is more uniform than during day-time. The distributions of the four pattern families magnify the day-night differences. Indeed, only $1 \mathrm{~V} \%$ exhibits no difference. On the contrary, 0V\% 

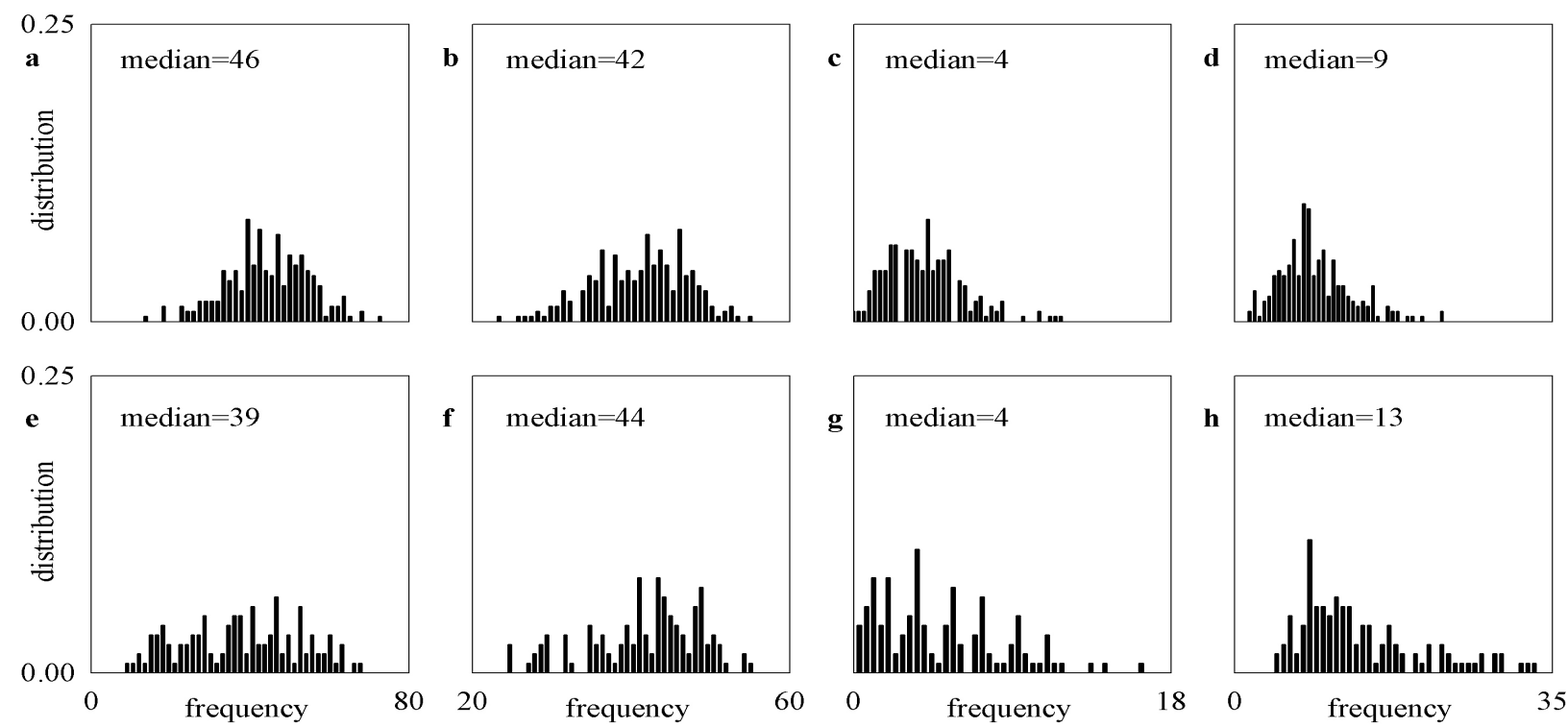

Figure 2. Day-time and night-time distributions of 0V\%, 1V\%, 2LV\% and 2UV\% during day-time (a,b,c,d) and nighttime $(\mathrm{e}, \mathrm{f}, \mathrm{g}, \mathrm{h})$ in a $\mathrm{N}$ subject.

strongly decreases during night-time, while $2 \mathrm{LV} \%$ and 2UV\% increase.

Fig. 2 shows the distribution of $0 \mathrm{~V} \%, 1 \mathrm{~V} \%, 2 \mathrm{LV} \%$ and $2 \mathrm{UV} \%$ derived from the entire $24 \mathrm{~h}$ heart rate variability series of a $\mathrm{N}$ subject during day-time $(\mathrm{a}, \mathrm{b}, \mathrm{c}, \mathrm{d})$ and nighttime $(e, f, g, h)$. The median of the distribution confirms that during day-time $0 \mathrm{~V} \%$ (a) is larger than during nighttime (e), while the opposite situation is observed in the case of $2 \mathrm{UV} \%$ (compare $\mathrm{d}$ with $\mathrm{h}$ ). In this example $1 \mathrm{~V} \%$ and $2 \mathrm{LV} \%$ seem to be unchanged (the medians in $\mathrm{b}$ and $\mathrm{c}$ are similar to those in $\mathrm{f}$ and $\mathrm{g}$ ).
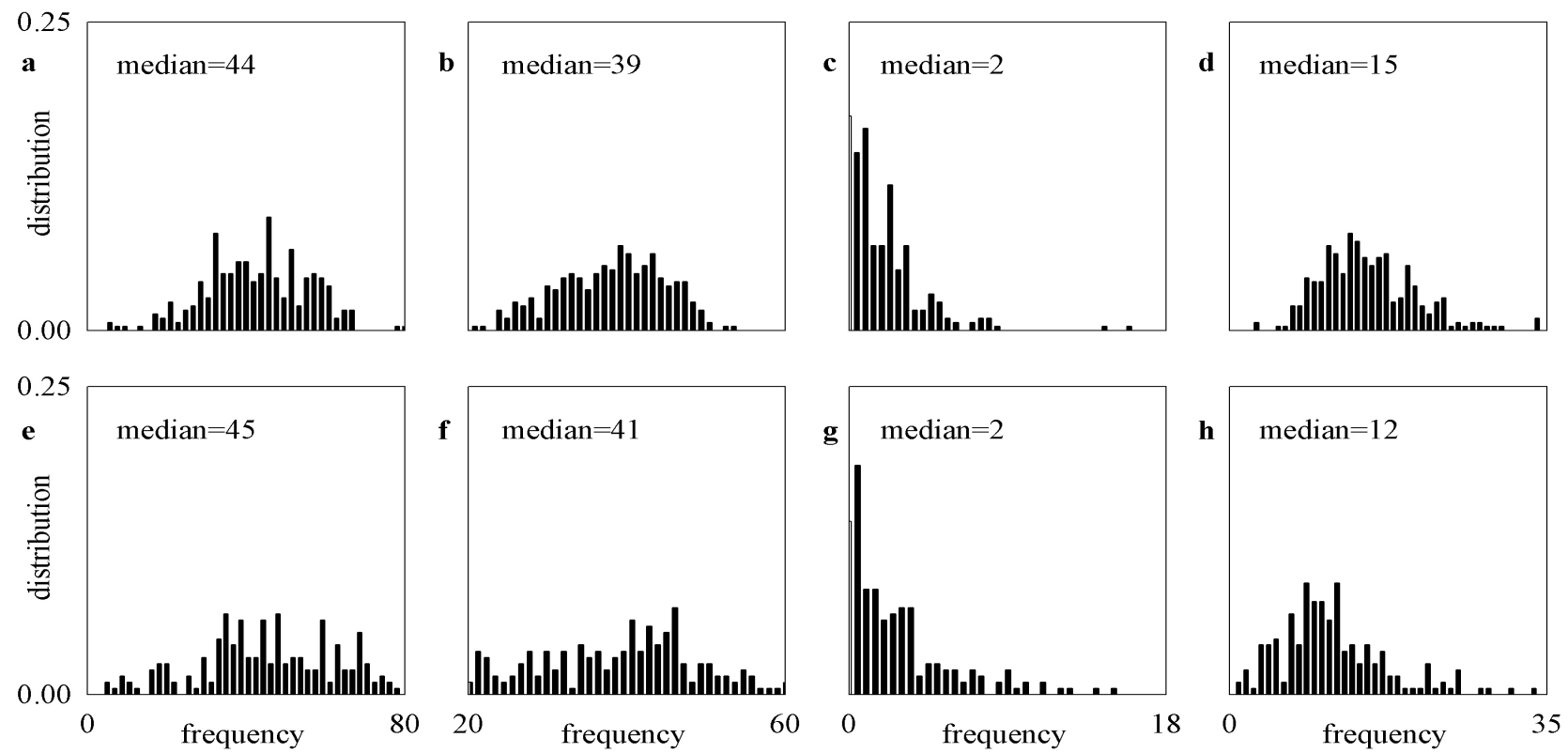

Figure 3. Day-time and night-time distributions of $0 \mathrm{~V} \%, 1 \mathrm{~V} \%, 2 \mathrm{LV} \%$ and $2 \mathrm{UV} \%$ during day-time (a,b,c,d) and nighttime $(\mathrm{e}, \mathrm{f}, \mathrm{g}, \mathrm{h})$ in a HF patient. 

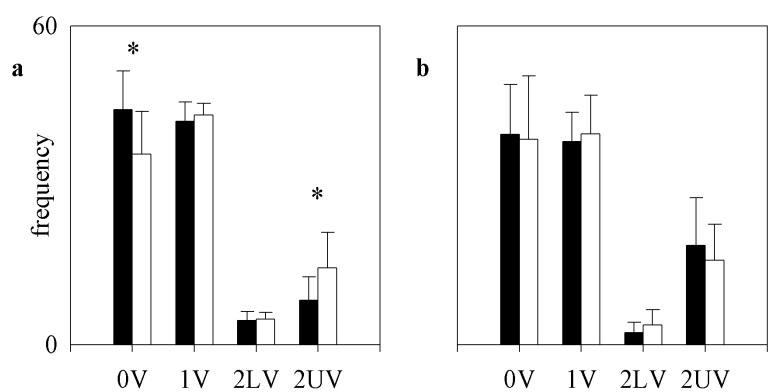

Figure 4. Grouped bar charts reporting the mean (+sd) of the median of the $24 \mathrm{~h}$ distributions of $0 \mathrm{~V} \%, 1 \mathrm{~V} \%$, $2 \mathrm{LV} \%$ and $2 \mathrm{UV} \%$ over $\mathrm{N}$ (a) and HF (b) populations. Solid and open bars are relevant to the results obtained during day-time and night-time respectively. The asterisk * marks a significant day-night difference.

night-time they were $35.87 \pm 8.05$ ( $\mathrm{p}<0.01$ night-time vs day-time), $43.26 \pm 2.20, \quad 4.82 \pm 1.23$ and $14.46 \pm 6.69$ ( $\mathrm{p}<0.05$ night-time vs day-time). In HF patients during day-time $\quad 0 \mathrm{~V} \%, \quad 1 \mathrm{~V} \%, \quad 2 \mathrm{LV} \%$ and $2 \mathrm{UV} \%$ were $39.63 \pm 9.40, \quad 38.29 \pm 5.45,2.30 \pm 1.89$ and $18.73 \pm 8.91$, while during night-time they were $38.68 \pm 11.98$, $39.69 \pm 7.27,3.68 \pm 2.93$ and $15.90 \pm 6.79$. In HF patients during day-time $2 \mathrm{LV} \%$ were significantly smaller and 2UV\% significantly larger than in $\mathrm{N}$ subjects, while during night-time no significant differences were observed between $\mathrm{N}$ and HF populations.

\section{Discussion}

This study confirms the ability of symbolic analysis applied to heart rate variability to distinguish different physiological conditions in $\mathrm{N}$ subjects $[3,4]$ even on $24 \mathrm{~h}$ Holter recordings. Indeed, in $\mathrm{N}$ humans during day-time, we found that $0 \mathrm{~V} \%$ is significantly larger than during night-time, while $2 \mathrm{UV} \%$ is significantly smaller. A similar finding was found in carefully controlled laboratory conditions during autonomic tests producing a sympathetic activation and a vagal withdrawal such as tilt and handgrip $[3,4]$. It seems that the number of stable patterns (i.e. 0V) tends to increase when sympathetic nervous system is more active and strongly modulate heart period duration, while unstable periods characterized by very rapid changes of the heart period and described by $2 \mathrm{UV}$ patterns are reduced. Therefore, in $\mathrm{N}$ subjects a clear circadian rhythmicity is detectable by indexes from symbolic analysis. As it occurs for the time and frequency domain indexes [5], this circadian rhythmicity is lost in the HF population. This finding confirms the capability of symbolic analysis to differentiate between normal and pathological populations [1] even on 24h Holter heart rate variability recordings. In addition, we detected another interesting difference between $\mathrm{N}$ and $\mathrm{HF}$ populations: during day- time the number of unstable patterns (i.e. 2UV) significantly increased in HF patients, while that of patterns with sustained changes (i.e. 2LV) significantly diminished. Increased electrical cardiac instabilities resulting in RR interval alternating patterns in HF patients might be responsible for this finding.

\section{Conclusions}

Heart rate variability analysis is performed on $24 \mathrm{~h}$ Holter recordings using indexes based on symbolic analysis (i.e. the rate of occurrence of four pattern families). These indexes identify two physiological conditions characterized by different cardiac autonomic regulations in healthy humans (i.e. day-time and nighttime) and differentiate healthy subjects from heart failure patients. The results prompt to include these indexes in the set of parameters that should be screened in standard 24h Holter analysis of heart rate variability. New studies on larger database should verify whether these indexes carry information independent of that derived from more traditional time and frequency domain indexes.

\section{References}

[1] Voss A, Kurths J, Kleiner HJ, Witt A, Wessel N, Saparin P. Osterziel KJ, Schurath R, Dietz. The application of methods of non-linear dynamics for the improved and predictive recognition of patients threatened by sudden cardiac death. Cardiovasc Res 1996;31:419-433.

[2] Wessel N, Ziehmann C, Kurths J, Meyerfeldt U, Schirdewan A, Voss A. Short-term forecasting of lifethreatening cardiac arrhythmias based on symbolic dynamics and finite-time growth rates. Phys Rev E 2000;61:733-739.

[3] Guzzetti S, Borroni E, Garbelli PE, Ceriani E, Della Bella P, Montano N, Cogliati C, Somers VK, Malliani A, Porta A. Symbolic dynamics of heart rate variability: a probe to investigate cardiac autonomic modulation. Circulation, 2005;112:465-470.

[4] Porta A, Guzzetti S, Montano N, Furlan R, Pagani M, Malliani A, Cerutti S. Entropy, entropy rates and pattern classification as tools to typify complexity in short heart period variability series. IEEE Trans Biomed Eng 2001;48:1282-1291.

[5] Malliani A, Pagani M, Lombardi F, Cerutti S. Cardiovascular neural regulation explored in the frequency domain. Circulation 1991;84:482-492.

Address for correspondence.

Alberto Porta, $\mathrm{PhD}$

Universita' degli Studi di Milano

Dipartimento di Scienze Precliniche (DiSP)

LITA di Vialba

Via G.B. Grassi 74

20157, Milan, Italy

E-mail: alberto.porta@unimi.it 